\title{
Transformación Del Sector Eléctrico Mexicano, Obstáculos Y Perspectivas De Competitividad Global En 2016
}

\author{
Agustín Vilchis Vidal, Doctor \\ Elsa Aranda Pastrana, Maestra \\ Universidad Autónoma de Ciudad Juárez
}

doi: 10.19044/esj.2016.v12n31p417 URL:http://dx.doi.org/10.19044/esj.2016.v12n31p417

\begin{abstract}
This paper discusses the transformation of Mexico's electricity sector at birth as a parastatal entity of the federal government in 1937, until it became a productive state enterprise in 2016. In this transit, a series of obstacles can be seen that define its insertion in a global energy environment complex, dynamic, and very competitive. The work method was mixed descriptive and longitudinal between 2008 and 2016, the assessment is based on national and international indicators of energy efficiency, fuel use, energy diversification, and human resources, as well as interviews with experts to learn about the competitive prospects of the sector. The results show that the productive state enterprise outstanding large cargo, such as labor liabilities, debts incurred in dollars and with variable interest rate, all affect their levels of competitiveness in an international and national environment. However, the accumulated knowledge of its technical staff, a culture of solid quality, diversification of its energy portfolio, coupled with a comprehensive infrastructure of power plants and the transmission network, envision scenarios that, if properly managed, can allow strategic alliances for cogenerate value in the electricity sector and can improve the competitiveness and sustainability indicators. However, it should also be mentioned that the exogenous context is unfavorable with a tendency to systematically reduce CFE's participation in the domestic market.
\end{abstract}

Keywords: Labor liabilities, specialized personnel, electric industry, and quality culture

\section{Resumen}

El presente trabajo aborda la transformación del sector eléctrico de México al nacer como una entidad paraestatal del gobierno federal en 1937, hasta ser una empresa productiva del estado en 2016. En este tránsito, se 
pueden observar una serie de obstáculos que definen su inserción en un ambiente mundial energético complejo, dinámico y muy competitivo. La metodología fue mixta de tipo descriptivo y longitudinal entre 2008 y 2016 , la evaluación es sustentada con base en indicadores nacionales e internacionales de eficiencia energética, uso de combustibles, diversificación energética y recursos humanos. Los resultados encontrados demuestran que la empresa productiva del estado carga grandes pendientes, como el pasivo laboral, las deudas contraídas en dólares y con tasa de interés variable, todo ello confina sus niveles de competitividad en un entorno internacional y nacional. Sin embargo, el conocimiento acumulado de su personal técnico, una cultura de calidad sólida, diversificación de su portafolio energético, aunado a una infraestructura integral de plantas generadoras de energía y la red de transmisión vislumbran escenarios que, bien gestionados, puedan permitir alianzas estratégicas para cogenerar valor en el sector eléctrico nacional y puedan mejorar los indicadores de competitividad y sustentabilidad. Sin embargo, también hay que comentar que el contexto exógeno es desfavorable con tendencias a disminuir sistemáticamente la participación de la CFE en el mercado nacional.

Palabras clave: Pasivo laboral, Personal especializado, industria eléctrica y cultura de calidad.

\section{Introducción}

El objetivo de este trabajo es analizar la transformación, retos y amenazas que ha enfrentado el sector eléctrico mexicano desde su nacimiento. United Nations Population Fund (2015), agencia de la Organización de las Naciones Unidas contabiliza la población mundial, indica que hay 7349 millones de personas, de las cuales corresponde el $26.1 \%$ al intervalo de 0-14 años, el 65.6\% al rango de 15-64 años, y el resto 8.3\% mayores de 65 años. Estos más de siete billones de humanos tienen diferentes características y exigencias de energía alrededor del mundo. Es decir, la diferencia se ve polarizada con el consumo de este servicio ya que el 75\% de la energía mundial la consume el 25\% de los países, principalmente desarrollados, en contra parte el $25 \%$ de la energía mundial la consume el 75\% de los países subdesarrollados (Quintanilla y Arfeuille, 2011).

En 2016 la recuperación de la economía mundial ha tenido un comportamiento heterogéneo, por un lado hay economías con crecimiento cercano al 1\% del producto interno bruto PIB, tal es el caso de los Estados Unidos de América (EUA), Alemania, Francia, entre otros (WB, 2016); mientras por el otro lado hay otras naciones como China e India con incrementos superiores al 5\% del PIB; también podríamos hablar de otra bloque de naciones cuyo crecimiento oscila entre el $2 \%$ al $4 \%$, por ejemplo 
México, Chile y Rusia (WB, 2016). Bajo esta premisa, los requerimientos energéticos mundiales sufren una transformación estructural que le da forma al sector. Lo anterior, exige nuevos mecanismos de cooperación a nivel internacional que proporcionen energía segura, asequible, eficiente, moderna y con menos emisiones de bióxido de carbono, acorde a las necesidades de los países, como lo expresa la Cumbre de las Naciones Unidas (COP21, ONU, 2015) y la Agencia Internacional de Energía (IEA, 2015).

Según el estudio de perspectivas de la energía mundial del periodo de 2015-2040 China e India serán los principales consumidores de energía, por ejemplo China en el 2030 consumirá más petróleo que EUA y en 2040 la demanda energética será casi el doble que de la de ese país, además consumirá gas equivalente a la Unión Europea. Por su parte, India requerirá 2.8 billones de dólares de inversión para suministro energético hasta 2040, en forma particular indica que será el segundo productor de carbono, después de China, pero también en el mayor importador de esa materia prima a partir de 2020. Aunque, su desarrollo debe de ser compatible con políticas ambientales muy restrictivas y el uso de tecnologías de punta que aumente su eficiencia (AIE, 2015).

Después de eso, las presiones internacionales por reducir los efectos del cambio climático el gas natural se perfila como el combustible con mayor crecimiento sostenido en el uso del sistema energético. No obstante, en la segunda década del siglo XXI las presiones por mantener un costo bajo son mayores, esto se explica por diversos factores, entre los más importantes: la indexación con los precios del petróleo, las políticas de eficiencia en su uso, la competencia de energías renovables, las inversiones diferidas, así como el uso del carbón. Por si esto no bastara, se prevé inversiones fuertes para poder llevar el gas, a través de gaseoductos, por largas distancias lo que eventualmente puede contribuir al incremento del costo de este combustible (AIE, 2015; SE, 2016).

De hecho, la inversión extranjera directa sigue una lógica de diversificación del riesgo e incremento de los beneficios, por ejemplo a nivel mundial los países desarrollados liderados por EUA, Francia y Alemania invirtieron entre 1990 y 2007 aproximadamente dos tercios de este rubro en el mundo, con un monto cercano a 11 trillones de dólares. En forma específica, en 2007 a la región de Latinoamérica le correspondió un 27\%, mientras que el 64\% Asía y el resto en Europa del este y África, el 9\% faltante a otras naciones (Salvatore, 2010).

Asimismo, el sector energético es estratégico para el desarrollo de cualquier país, en el caso del sector eléctrico en México requiere de grandes inversiones para poder atender los requerimientos de los diferentes actores productivos. Aunado a ello, la imposibilidad de poder absorber los costos financieros de la reconversión de sus diferentes plantas generadoras a ciclo 
combinado, así como mejorar las líneas de transmisión y el pasivo laboral, dificulta cumplir con las expectativas de una institución sana financieramente hablando. En palabras de Sosa Pavón (2008), considera que el gobierno mexicano debe buscar mecanismos de inversión que permitan aliviar y evitar el rezago del sector eléctrico, por ejemplo él menciona que mientras en 1987 este sector la sociedad demandaba 96.3 Tera watt-hora, para 2010 ascendió a 362 Tera watt-hora y con proyecciones para 2020 alrededor de los 500 Tera watt-hora. En esta misma lógica, se alinea Willars (2008) al mencionar que el indicador de intensidad energética (consumo de energía entre el producto interno bruto del periodo), indica el aumento de la cantidad de energía requerida para producir bienes y servicios. Lo anterior, refleja la mayor importancia relativa de los sectores industriales intensivos para hacer sus actividades requieren electricidad.

Ante este panorama, el objetivo de este artículo es reflexionar sobre el uso racional de la energía, en general, pero de la electricidad en particular, el rol de las autoridades gubernamentales, principalmente: Secretaría de Hacienda y Crédito Público, Secretaría de Energía y Comisión Federal de Electricidad, así como la Comisión Reguladora de Energía y el Centro Nacional de Control de Energía, en la transición del sector, además analizar los obstáculos y perspectiva que definen su inserción en un ambiente mundial energético complejo, dinámico y muy competido. Para lograrlo, este documento se integra de antecedentes, la competitividad y el sector eléctrico, metodología, resultados y discusión.

Para ello, tienen grandes retos que superar, entro otros se encuentra la adecuación de las tarifas, la incorporación de las alianzas público-privadas que permitan nuevos mecanismos de funcionamiento que fortalezca el crecimiento sano del país (Quintanilla y Arfeuille, 2011).

\section{Antecedentes}

A nivel mundial se está realizando una transición energética hacia energía renovable y nuevas tecnologías, para el 2025 se propone que el 25\% de la energía sea renovable (WEF, 2015). En el caso particular de México, los escenarios se basan en el Plan Nacional de Desarrollo 2012-2018, así como en el Programa del Sector de Energía, los cuales incluyen economía competitiva y sustentabilidad ambiental; además el Programa de Desarrollo del Sistema Eléctrico Nacional (PRODESEN 2015-2030), así como el Programa de Ciencia y Tecnología (PECYT), con el fin de hacer efectiva la ciencia, tecnología e innovación en el desarrollo nacional.

La misión de la Secretaría de Energía es: "garantizar el suministro competitivo, suficiente, de alta calidad, económicamente viable y ambientalmente sustentable de energéticos para el desarrollo de la vida 
nacional” (SE, 2016). Para ello, desde su creación en $1994^{56}$, ha sufrido una serie de transformaciones reglamentarias, por ejemplo en 1997 se publicaron en el Diario Oficial de la Federación reformas y adiciones a la reestructuración y redimensionamiento de las funciones de la dependencia; en 2001 hay un enfoque de especialización en hidrocarburos y electricidad con la creación de diferentes subsecretarías de estado y en el 2004 hay un proceso de reestructuración laboral hacia una organización más horizontal.

No obstante, los cambios más significativos se dan en 2012 con una reformulación de áreas y facultades de funcionarios, en 2013 se da el proceso de transición de la reforma energética, con el Decreto para modificar los artículos 25, 27 y 28 constitucionales y la inclusión de 21 transitorios. Al año siguiente, se publican 21 leyes secundarias, 24 reglamentos y 1 ordenamiento y de manera adicional en 2015 se publica la Ley de Transición Energética. Como resultado de esto, se crea: el Fondo Mexicano del Petróleo (FMP, administra ingreso por contratos y asignaciones de actividades de exploración y extracción de petróleo); la Comisión Nacional de Hidrocarburos (CNH) y la Comisión Reguladora de Energía (CRE), las últimas dos instituciones tienen independencia técnica y administrativa, cuyas funciones son regular la participación de empresas públicas y privadas para asegurar el funcionamiento eficiente de los energéticos.

En esta misma lógica, se crearon otros dos organismos públicos descentralizados: El Centro Nacional de Control de Energía (CENACE, controla la operación del Sistema Eléctrico Nacional y el mercado eléctrico mayorista) y el Centro Nacional de Gas Natural (CENEGAS que administra el sistema de transporte y almacenamiento de gas natural). Es conveniente destacar que en 2015 se presenta el Programa de Desarrollo del Sistema Eléctrico Nacional (PRODESEN 2015-2030).

Con respecto a la protección ambiental, se crea la Agencia de Seguridad, Energía y Ambiente (ASEA la cual supervisa la seguridad industrial y la operación de empresas petroleras), además la Comisión Nacional para el Uso Eficiente de la Energía (CONUEE que promueve prácticas de eficiencia y aprovechamiento sustentable de la energía). De manera adicional, se hacen algunas modificaciones organizacionales de diversos institutos afines al sector energético, en particular destaca el cambio del Instituto de Investigaciones Eléctricas a Instituto Nacional de Electricidad y Energías Limpias, cuya incorporación adicional tiene un enfoque a la prevención de la contaminación en la industria eléctrica (DOF, 2016).

\footnotetext{
${ }^{56}$ La Secretaria de Energías, Minas e Industrias Paraestatales cambia su nombre a Secretaría de Energía. (DOF, 1994).
} 


\section{Diseño metodológico}

Pacheco (2006) entiende la investigación científica como una indagación sistemática y metódica, que con base en un conocimiento objetivo y previo ordenado en una plataforma teórica, busca agregar conocimiento, dilucidando y transformado la realidad. Hernández y otros (2005), conceptualizan el proceso del diseño de la investigación como el plan para obtener información, que permita dar respuesta a la pregunta de investigación y cumplir los objetivos del estudio. En forma similar, Beltrán y Alvira (2000) indican que el investigador requiere de un plan que guíe el proceso de recoger, analizar e interpretar datos y observaciones, además se deben de dar respuestas claras y no ambiguas a los cuestionamientos iniciales.

Esta investigación longitudinal (2006-2016) se fundamenta en la revisión de documentos que permiten un análisis cuantitativo y cualitativo, este último enfoque se refuerza con entrevistas con algunos líderes estratégicos de la Comisión Federal de Electricidad y de organismos paraestatales del sector (Coordinación de Proyectos Termoeléctricos, Gerencia Regional de Producción Norte, Instituto de Investigaciones Eléctricas). Esta investigación de tipo descriptivo ofrece un panorama de la competitividad del sector eléctrico y las transformaciones que ha sufrido, particularmente, después de la reforma energética de México. A continuación se muestra la definición conceptual y operativa de las variables cuantitativas y cualitativas de esta investigación. Ver Tabla 1.

Tabla 1: Operación de variables: Competitividad y transformaciones del sector eléctrico mexicano

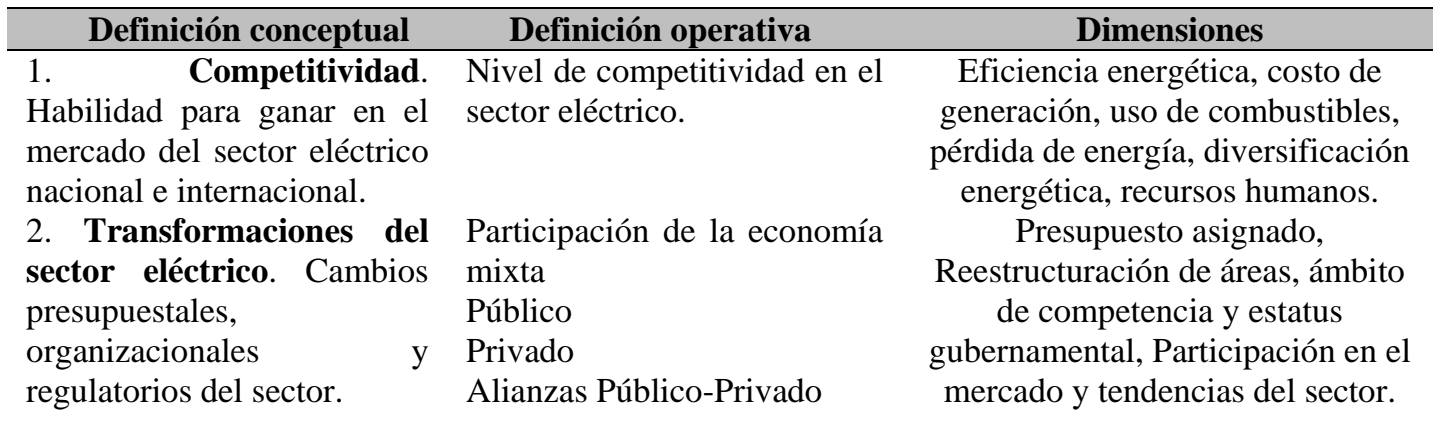

Fuente: Elaboración propia, 2016

Una vez realizada la conceptualización de las variables, es necesario definir las técnicas de recopilación de la información, así como las técnicas de análisis de los datos. Para ello, se puede observar el esquema de investigación que permite establecer las etapas de investigación y los instrumentos y mecanismos metodológicos, así como la estructura del diseño de la investigación. Ver Figura 1. 
Figura 1. Diseño Metodológico de la Investigación del Sector Eléctrico Mexicano

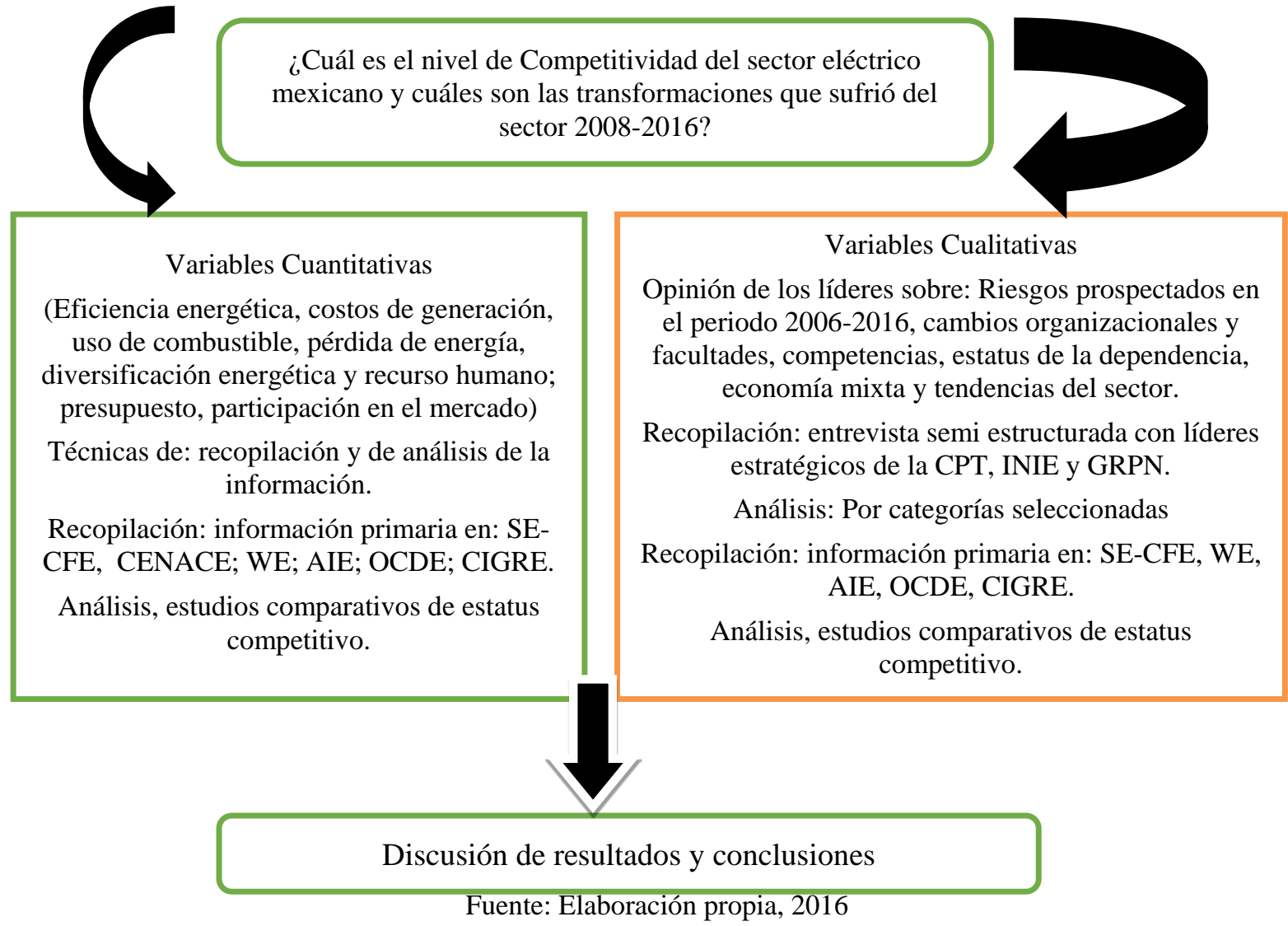

\section{Resultados de la competitividad del sector eléctrico mexicano}

Quintanilla y Arfeciille (2011), comentan que a partir del cambio jurídico de la Ley del Servicio Público de Energía Eléctrica en México en 1992, la participación del sector privado ha crecido en forma sistemática, el primer permiso denominado Mérida II tuvo una capacidad de 532 Mega-watts, para 2004 la capacidad autorizada fue de 1,255 Mega-watts. Es importante apuntar que, el Banco Mundial en 2006 aportó setenta millones de dólares a productores independientes de la Comisión Federal de Electricidad para generar electricidad a través de energías renovables, en 2011 la participación del sector privado en la generación del sector eléctrico ascendió a 21,468 Mega-watts, lo que equivale al $46.5 \%$ de la energía renovable en el país.

Estos mismos autores, alertan sobre replantear el concepto de desarrollo y no anteponer o priorizar el desarrollo económico sobre el desarrollo humano, moral, mental y cultural de una sociedad. En otras palabras, el crecimiento de una economía requiere de una visión global e integral que permita armonizar el desarrollo con un planteamiento incluyente, para evitar 
el incremento de las desigualdades y la pauperización de las regiones más desprotegidas.

Si se toma el caso de dos unidades estratégicas de la Comisión Federal de Electricidad, la Coordinación de Proyectos Termoeléctricos (CPT); la Gerencia Regional de Producción Norte (GRPN) y una dependencia paraestatal alineada a la Secretaria de Energía, que le proporciona servicio de investigación y desarrollo al sector energético del país, en particular al sector eléctrico, el denominado Instituto de Investigaciones Eléctricas (IIE). ${ }^{57}$ Con estas tres dependencias, se plantea una aproximación al nivel de competitividad de la Comisión Federal de Electricidad (CFE) en el sector eléctrico internacional y nacional que permite abordar los obstáculos y las perspectivas de competitividad global en el periodo 2008-2016.

La investigación tecnológica es un proceso lento, al (IIE) le costó 25 años ser reconocido como Centro Público de Investigación, bajo los criterios del marco jurídico de la Ley de Ciencia y Tecnología. El IIE nace en 1975 como un organismo descentralizado de la Administración Pública integrante del sector energético; en la mitad de la segunda década del siglo XXI cultiva líneas de investigación y desarrollo tecnológico para ofrecer respuestas a los requerimientos tecnológicos y de innovación al sector eléctrico y energético del país (IIE, 2009).

En una segunda etapa, en la década de los ochenta y acorde al proceso de estabilización económica, coadyuva en la apertura comercial y el crecimiento de las exportaciones mediante el ajuste de sus líneas de investigación que produce una dinámica de crecimiento. Como referencia, en ese periodo nacen las empresas con base tecnológica y comercialización de la propiedad intelectual del Instituto.

En una tercera época, en los noventas se da énfasis en la transferencia tecnológica, lo que conlleva a incrementar el personal y la infraestructura; aunado a lo anterior se crea una empresa de innovación tecnológica, además fortalece los lazos comerciales con CFE y Petróleos Mexicanos (PEMEX). El IIE sobresale por su nivel de autosuficiencia financiera con una $74.4 \%$, aunque también habría que decir que de este porcentaje sólo el 11\% proviene de clientes no gubernamentales. Sin embargo, al sólo contar con dos clientes claves como CFE y PEMEX (ambos generan el 66\% de los ingresos), cabe mencionar que omiten consolidar una cobertura de mercado a nivel global, no obstante, tienen ingresos mínimos provenientes de servicios a naciones de Centro y Sudamérica. Es en este punto, donde se dan pasos sólidos para mejorar la autosuficiencia financiera, al pasar de 20 a cerca del $80 \%$ de sus

\footnotetext{
${ }^{57}$ El Instituto de Investigaciones Eléctricas fue estudiado en 2009, pero en 2016 cambia su nombre a Instituto Nacional de Electricidad y Energías Limpias (DOF, 2016).
} 
recursos. Como ejemplo, se le encarga el estudio de costo beneficio del horario de verano.

El cuarto periodo, abarca la primera década del presente siglo, logra ser reconocido como Centro Público de Investigación del Consejo Nacional de Ciencia y Tecnología (CONACYT) (DOF, 2003). Lo anterior, en una lógica de globalización, acreditaciones, certificaciones, logran consolidar sus procesos de sistema de gestión de calidad, acreditación y certificación de instalaciones y personal, de manera adicional pasan mecanismos de evaluación de proveedores con calificativos de confiables. Es importante destacar que en 2009 la facturación total de esta dependencia ascendía a 650.4 millones de pesos (IIE, 2010). Por otro lado, se fomenta la internacionalización del instituto y de sus investigadores, los cuales pertenecen a diversas organizaciones mundiales del sector, tales como la Agencia Internacional de Energías (AIE); el Consejo Mundial de Energía (WEC), y el Consejo Internacional de Grandes Sistemas Eléctricos (CIGRE) (IIE, CFE, 2010).

Al mismo tiempo, el CONACYT, al pertenecer el IIE como centro público de investigación ha sido evaluado con la denominación Excelente $(91-100 \%)^{58}$. Pese a ello, el IIE en el documento "El Plan Estratégico Institucional 2009-2013” contempló en su análisis de riesgos para este periodo tres elementos fundamentales ${ }^{59}$. El primero es la restricción presupuestal federal (alta probabilidad y alto impacto); el segundo la mayor participación de productores independientes con su propia tecnología (alta probabilidad e impacto medio); el tercero, políticas de CFE para redirigir aportaciones para Investigación \& Desarrollo (media probabilidad y alto impacto). En este sentido, se coincide con Luhmann (1998) cuando habla de que el riesgo es una forma de realizar descripciones presentes del futuro, que decide una alternativa, en función de los riesgos contemplados.

La quinta fase, corresponde a la segunda década del siglo XXI, vive nuevos retos, en el marco de la reforma energética se transforma de IIE a Instituto Nacional de Electricidad y Energías Limpias (INEEL) (DOF, 2016). En concordancia con la ejecución de la reforma energética, este centro de investigación es integrado a la Secretaría de Energía, entre sus funciones sustantivas se consolida la promoción y difusión criterios y de metodologías, además del desarrollo de tecnologías para la prevención de la contaminación

58 Evaluación de convenios de Desempeño de Centros Públicos de Investigación, los criterios incluyen indicadores de publicaciones en revistas arbitradas, ponencias internacionales, patentes, derechos de autor, personal de investigación con posgrado y autosuficiencia financiera, entre otros. CONACYT (2010).

${ }^{59}$ El Plan Estratégico Institucional 2009-2013 fue un ejercicio retrospectivo que consideró el Modelo de Evaluación Numérica de Riesgos de la Secretaría de la Función Pública, del Órgano Interno de Control. 
en la industria eléctrica (INEEL, 2016). Ahora mismo, en 2016, los riesgos proyectados en el ejercicio 2009-2013 se concretaron, lo que ha generado una serie de contratiempos y oportunidades para este Instituto, dentro de estos cambios está el transformarse a Instituto Nacional de Electricidad y Energías limpias (DOF, 2016).

En esta última etapa, hay que poner mayor atención, porque el decreto y la Ley Orgánica de la Administración Pública Federal, específicamente el artículo 33 (DOF, 2008), el cual establece las funciones de rectoría de la Secretaría de Energía en materia de planeación energética a mediano y largo plazo. Hasta el momento, no se tiene elementos que permitan, de manera definitiva, emitir un juicio con fundamento para observar el estatus de la soberanía y seguridad energética, así como la mayor productividad energética, la reducción de impactos ambientales de la producción y consumo de energía, mayor participación de las energías renovables, sobre todo satisfacción de las necesidades energéticas básicas de la población, sobre todo la de menores condiciones socioeconómicas.

La segunda dependencia de estudio es la Coordinación de Proyectos Termoeléctricos (CPT) que es la encargada de incrementar la capacidad de generación de energía eléctrica acorde a los proyectos estratégicos de la CFE y a lo definido en el Programa de Obras e Inversión del Sector Eléctrico (POISE), además de participar en la rehabilitación y modernización de la infraestructura existente, lo anterior ha permitido entre 2001y 2008 incorporar 12,190 Mega-watts con una participación activa del 92.14 de dicha CPT, a través de 28 centrales Termoeléctricas, una central Eolo eléctrica, y cuatro conversiones a Ciclo Combinado y en la repotenciación de la Central Núcleo Eléctrica de Laguna Verde con un incremento de $261 \mathrm{MW}$. Entre 2008 y 2010 se construyeron cinco proyectos termoeléctricos que incrementaron la capacidad en 1,813 Mega-watts.

Aunado a lo anterior, se gestaron en este periodo proyectos de cogeneración de energía con PEMEX, y los proyectos de las terminales de Gas Natural Licuado, en un primara instancia la Terminal de Gas Natural Licuado (TGNL) de Altamira Tamaulipas, para posteriormente la TGNL de Manzanillo, Colima, como respuesta a la tendencia de utilizar más gas en la generación de energía eléctrica, hasta esa primera década del siglo XXI, la mayoría del gas era de importación vía Texas, EUA. (CPT, 2008)

Otra categoría influyente, es el tipo de tecnología utilizada, para 2008 el 73.18\% de la capacidad instalada en el país era mediante las termoeléctricas con el uso de combustible fósil (Centrales de Ciclo Combinado, Termoeléctricas, Centrales de Carbón, Turbo Gas y Combustión Interna), contra el 26.82\% con tecnologías de generación que incluye Hidráulica, Geotermia, Nuclear y Eólica, entre otras. Asimismo, en estudios prospectivos de la CFE en general, pero de la CPT en particular, entre 2008- 
2017 se vislumbra el uso del gas natural como el combustible con mayor potencial para la generación eléctrica, lo anterior se confirma que entre el 2014 y 2015 se adjudicaron siete proyectos estratégicos para entrar en operación entre 2017 y 2018 y llevar gas a diferentes regiones del país, principalmente de EUA a diferentes regiones del país, (noreste, noroeste, centro y occidente), por sus beneficios económicos y ambientales (CENEGAS, 2016)

Para poder hacer posibles estos proyectos, la CFE y la CPT en 2008 y actualmente, tienen tres mecanismos de financiamiento: el primero de ellos es PIDIRIEGAS ${ }^{60}$ (deuda de largo plazo); Obra Pública Financiada (OPF), recurso de CFE pero con créditos externos, y productores externos de energía PEE, básicamente las dos primeras subastas de 2016 de la CFE.

Por tal razón, la CPT su función sustantiva es supervisar el cumplimiento de los contratos de los proveedores que garantice el apego estricto a las normas técnicas, técnicas de calidad, ambiental y de seguridad, así como el adecuado desarrollo de la ingeniería de detalle, la construcción puesta en servicio y la entrega de centrales confiables. Vale la pena destacar que, empresas de clase mundial han participado en el proceso de construcción de instalaciones eléctricas, por ejemplo: Siemens, General Electric, Mitsubishi e Iberdrola, entre otras (CPT, 2008). Por otro lado, la CPT, con sus estudios prospectivos 2008-2017 pretende que la generación de energía renovable alcance el $20 \%$, contra el $80 \%$ con la tecnología de ciclo combinado (CPT, 2008-2017).

Otro eje rector sustantivo de la CPT, sin duda es la preocupación de la situación ambiental, por esto se han promovido proyectos de repotenciación y modernización de las instalaciones eléctricas con el fin de disminuir el impacto ecológico. Por ejemplo, Punta Prieta en la Paz, Baja California Sur, se eliminó el 80\% de óxidos de nitrógeno-azufre (NOx-SOx), así como otras transformaciones en la central del valle de México con $40 \%$ de disminución de NOx (CPT, 2008).

Antes de la reforma energética, del 2014 al 2016 la CPT tenía contemplado el incremento de generación eléctrica a 22,771 Mega-watts, conforme al estudio prospectivo del POISE (2008-2017), en esta planeación la CPT contribuiría con el $88.14 \%$ de esa capacidad. Lo anterior, es un síntoma de la pérdida de presencia en cuatro puntos porcentuales al pasar de 92.14 en 2001-2006 al 88.14 en 2008-2017.

En otro orden de ideas, el comportamiento a nivel mundial de fuentes alternas en el mundo, sobresale la disminución progresiva del uso del petróleo de 5.4 a 5\% entre 2003-2015; en ese mismo periodo, el carbón paso

${ }^{60}$ Proyecto de Infraestructura Productiva de Largo Plazo, CFE. 2015 
de 41.4 a 37.6\%; la energía nuclear de 16.9 a 12.9\%; pero el incremento de dos tipos de combustibles: gas de 17.5 a $25.7 \%$; las energías renovables del 18.7 al 18.8\%. En el caso particular de México, tiene contemplado para 2015 alcanzar el 25\% de su capacidad generadora mediante energía limpia (CPT, 2008, AIE, 2015).

Por su parte, la Subdirección de Generación (SDG) es la encargada de la generación de energía eléctrica a través de cinco gerencias que se dividen el territorio de México. De manera particular, en 2010 la capacidad instalada en todo el territorio fue de 49,811.02 Mega-watts, destaca que los productores externos de energía generaron el 23.9\% con 11,906.9 Mega-watts, mayor que la región Noroeste y Central juntas, con 5,848.26 y 5562.08 Mega-watts respectivamente. Vale la pena destacar que, la región Sureste con 1,0277.84 es la región que mayor capacidad instalada tiene una participación de una quinta parte de la generación nacional (CFE, 2010), tal como se observa en la Tabla 2.

Tabla 2. Capacidad efectiva instalada en México, 2010.

\begin{tabular}{lc}
\hline Gerencia & Capacidad en Mega-watts \\
\hline Noroeste & $5,848.26$ \\
Norte & $6,826.44$ \\
Occidente & $9,270.99$ \\
Central & $5,562.08$ \\
Sureste & $10,277.84$ \\
Plantas móviles & 118.51 \\
Productores Externos de Energía & $11,906.9$ \\
Total & $\mathbf{4 9 , 8 1 1 . 0 2}$ \\
\hline
\end{tabular}

Fuente: CFE, 2010

De manera particular, la tercera área estudiada es la Gerencia Regional de Producción Norte, GRPN (para efectos de la CFE, ubica estados de la región norte y noreste del país, comprende Chihuahua, Durango, Coahuila, Nuevo León y la parte norte de Tamaulipas), en su reflexión estratégica lo contemplaba en 2010 al mencionar que los productores independientes tienen centrales de generación en todos los estados de esta gerencia. Tan es así que, que la tecnología utilizada en sus instalaciones es de ciclo combinado, con innovaciones de vanguardia y una capacidad instalada de 3,395.50 Mega-watts que representa el 49.71\% en la participación de mercado en la región norte, entre las zonas más industrializadas de México (GRPN, 2010).

Para hacer frente a esta competencia, el gerente comenta que: "un diferenciador clave es la tecnología de información y comunicación con la que cuenta la CFE, en general y la GRPN en particular, además del Sistema Integral de Gestión, multisitios, ambos sistemas no los tienen los productores independientes”. Aunado a ello, cuentan con una masa crítica laboral de un 28.1\% de colaboradores con carrera universitaria y con posgrado (25\% 
licenciatura, 3\% maestría y 0.1\% doctorado), aunque un poco más de un tercio tiene sólo educación básica (GRPN, 2010).

Además, otro elemento clave que ayuda a enfrentar la competencia de productores independientes es la reconversión de las centrales y el mantenimiento, además de las nuevas construcciones de instalaciones de generación de electricidad. Sin embargo, hay que mencionar que depende del Plan Nacional de Desarrollo y del Programa de Obras e Inversión del Sector Eléctrico (POISE), de 2011-2025 que pretende un incremento de la capacidad a $37655 \mathrm{MW}$, lo que equivale a un $75.59 \%$ de la capacidad de 2010 (POISE, 2011-2025).

No obstante lo anterior, es importante resaltar que la Gerencia Regional de Producción Norte ha mantenido su disponibilidad energética ${ }^{61}$ entre 85 y 89\% en el periodo 2008-2010, que al compararlo con el estándar internacional de 86.5\% (NER, USA), lo ubican entre las mejores regiones productoras a nivel nacional (GRPN, 2010). Asimismo, los esfuerzos por proteger el miedo ambiente ha tenido resultados alentadores, por ejemplo las emisiones de bióxido de carbono que fueron evitadas han crecido de $15.7 \mathrm{mil}$ toneladas en 2009 a 20.1 mil toneladas en 2012, cuyo logro se debe a tecnologías limpias y diversificación en el combustible. Ver tabla 3.

Tabla 3 Instalaciones de la Gerencia Regional de Producción Norte en 2010

\begin{tabular}{lcl}
\hline Tipo de central & Número & Combustible \\
\hline Termoeléctricas & 2 & Combustóleo \\
Ciclo combinado & 4 & Combustóleo y Gas \\
Turbo gas & 6 & Gas \\
Hidroeléctricas & 6 & Agua \\
Carboeléctricas & 3 & Carbón \\
Total & 21 & \\
\hline
\end{tabular}

Fuente: GRPN, CFE, 2010

En contraste para 2015, la Subdirección de Generación ejerció un presupuesto de inversión de 17,391 millones de pesos, que representa una reducción de 1.69\% respecto del presupuesto de 2014 de 17,690 millones de pesos (CFE, 2015). Pese a ello, la aplicación de la inversión a mantenimiento fue superior en 6.8\% contra 2014, al pasar de 8,259.4 a 8,823.4 millones de pesos, con esto las plantas continuaron operando de manera oportuna. En la siguiente tabla se observa el comportamiento del sistema eléctrico nacional y la creciente participación de los productores externos independientes, así como el índice de pérdidas de energía entre 2004-2014.

\footnotetext{
${ }^{61}$ Representa el porcentaje de energía disponible referida a la generación teórica durante un periodo determinado, es decir es la facultad que posee dicha unidad para aportar su generación al sistema eléctrico. NER, USA, 2010.
} 
Tabla 4 Evolución del Sistema Eléctrico Nacional 2004-2014 (GW-h)

\begin{tabular}{ccccccc}
\hline Concepto & $\mathbf{2 0 0 4}$ & $\mathbf{2 0 0 6}$ & $\mathbf{2 0 0 8}$ & $\mathbf{2 0 1 0}$ & $\mathbf{2 0 1 2}$ & $\mathbf{2 0 1 4}$ \\
\hline Total & 217,793 & 235,471 & 247,369 & 256,402 & 278,086 & 284,695 \\
Público & 208,634 & 225,079 & 235,871 & 242,538 & 261,894 & 260,138 \\
Importación & 47 & 523 & 351 & 397 & 2,166 & 2,124 \\
& $0.021 \%$ & $0.22 \%$ & $0.141 \%$ & $0.154 \%$ & $0.77 \%$ & $0.746 \%$ \\
Privado & 9,112 & 9,869 & 11,147 & 13,467 & 14,025 & 22,433 \\
& $4.18 \%$ & $4.19 \%$ & $4.5 \%$ & $5.25 \%$ & $5.04 \%$ & $7.87 \%$ \\
Pérdidas & 34,901 & 39,600 & 41,409 & 44,252 & 44,050 & 41,321 \\
& $16.02 \%$ & $16.81 \%$ & $16.73 \%$ & $17.25 \%$ & $15.84 \%$ & $14.51 \%$ \\
\hline
\end{tabular}

Fuente: Adaptación, SENER, 2015

Por otro lado, El CENACE, en noviembre de 2015, emitió las bases para la licitación para contratos de largo plazo de cobertura eléctrica para la compraventa de potencia, energía eléctrica acumulable (quince años) y certificados de energías limpias (veinte años); en abril de 2016 se dio el fallo que abarcó once empresas ganadores con 69 participantes de 227 ofertas, que se relacionan a continuación, Ver Tabla 5.

Tabla 5. Monto de inversión por origen en la primera subasta de CFE, 2016

\begin{tabular}{cc}
\hline País & Monto de inversión \% \\
\hline Italia & 41.64 \\
México & 36.36 \\
China & 9.3 \\
China-México & 5.1 \\
EUA & 4.98 \\
Canadá & 2.61 \\
Total & $100 \%$
\end{tabular}

Fuente: CENACE, 2016

En conjunto, logró atender una demanda de 5,389 millones de certificados de energías limpias CELs ${ }^{62}$, además 5.402 millones de Megawatts hora de energía, equivalente a casi un 85\% de la demanda inicial de la

${ }^{62}$ Tendrán derecho a recibir Certificados de Energías Limpias (CEL) por un período de veinte años los Generadores Limpios que representan a: I. Centrales que entren en operación con posterioridad al 11 de agosto de 2014; II. Las Centrales Eléctricas Legadas que generen energía eléctrica a partir de Energías Limpias que hayan entrado en operación antes del 11 de agosto de 2014, siempre y cuando hayan realizado un proyecto para aumentar su producción de Energía Limpia; III. Las Centrales Eléctricas Limpias que cuenten con capacidad que se haya excluido de un Contrato de Interconexión Legado a fin de incluirse en un Contrato de Interconexión en los términos de la Ley, durante el periodo en el que el titular del contrato cuente con el derecho de incluir dicha capacidad en el Contrato de Interconexión Legado. Se recibirá un CEL por cada Mega-watts-hora cuando no se use combustible fósil; cuando se use combustible fósil un CEL por cada Mega-watts-hora generado en las Centrales Eléctricas Limpias que representen, multiplicado por el porcentaje de energía libre de combustible, con base en la metodología propuesta por la Comisión Reguladora de Energía, CRE. Diario Oficial de la Federación, 2014. 
CFE. Destaca, la participación de Italia y México, con un porcentaje conjunto del 78\%, aunque hay que mencionar la poca participación de los socios comerciales regionales de México, con solo el 7.59\% (CENACE, 2016).

En una segunda subasta, en septiembre de 2016, los resultados fueron favorecidas 23 empresas, con 56 plantas, con una distribución tecnológica dominada por Solar Fotovoltaica con 37 plantas que representa 58.9\% de las centrales subastadas, seguidas con tecnología Eólica con el17.8\%, con igual porcentaje del 10.7\% coinciden el Ciclo Combinado e Hidroeléctrica y con sólo el $1.7 \%$ la tecnología de Geotermia. Los resultados engloban 8.9 millones de Mega-watts de energía; 9.3 millones de CEL y mil 187 Megawatts-año de Potencia, cubriendo entre 80 y $87.2 \%$ de lo solicitado por la CFE. En conjunto, la capacidad de generación de energía es de 3 mil 776 Mega-watts en total, de los cuales tres cuartas partes de esta producción se realizara en instalaciones nuevas (CENACE, 2016).

Es importante destacar que, la Comisión Federal de Electricidad fue adjudicada con dos instalaciones, la primera para la región del Occidente y la única con tecnología de Geotermia con una capacidad de solo 25 Megawatts, aunque destaca la central con tecnología de Ciclo Combinado para la región Noroeste, en Cananea, con una capacidad de 394.1 Mega-watts. Con esto, se observa que la CFE solo contribuye con el $3.6 \%$ por el número de infraestructuras y sería la segunda con mayor capacidad, únicamente por debajo de las instalaciones generadoras en la región Noreste, en Reynosa con 505 Mega-watts (CENACE, 2016).

En cuanto al número de unidades generadoras aprobadas, destaca la empresa Alten Energías Renovables México Cuatro con seis plantas, todas en la región Occidente Centro, en Aguascalientes, con una capacidad de 150 y 140 Mega-watts en conjunto, con tecnología Solar Fotovoltaica. Con una segunda posición, está la empresa Frontera México Generación S de R.L. de C.V con cinco unidades, para la región Noreste, particularmente en Reynosa, cuya tecnología de generación es de Ciclo Combinado y con una capacidad de 505 Mega-watts en cada una de las instalaciones (CENACE, 2016).

\section{Discusión de resultados}

El aumento de la cantidad de energía requerida para producir bienes y servicios en una nación, refleja la mayor importancia relativa de sectores industriales intensivos en el uso de dicha energía. (Willars, citado en Bauer y Colín, 2008). En el caso particular de México, el crecimiento de la demanda de energía ha crecido alrededor del 4.8\% anual (CFE, CPT, 2006-2016), contra un 2.7\% anual promedio del consumo mundial (WEC, 2016), mientras que la economía mexicana crece a tasas inferiores del $2.52 \%$ en la última década (WB, 2016). Algunos especialistas sugieren que el sector 
eléctrico requiere inversiones del último decenio (2006-2016) de cerca de 6 mil millones de dólares (Rodríguez, 2006), ante esto, Sosa (2008, citado en Bauer y Colín) comenta que la inversión mayúscula del sector eléctrico no puede ser absorbida por el gobierno federal. Por esta razón, el poder ejecutivo debe buscar mecanismos de inversión que permitan aliviar y evitar el rezago del sector.

Sin embargo, también debe de considerarse el riesgo que genera el crecimiento económico, Beck (2002) en su tipología de los riesgos globales hace énfasis en la destrucción ecológica y peligros tecnológico-industriales motivados por la riqueza, efecto invernadero, solo por mencionar algunos. Él, señala que el asumir la objetividad de los peligros globales es potenciar la construcción de instituciones transnacionales con el objetivo de llevar una política de desarrollo sostenible.

Para Goldstein (2009), considera la complejidad de intereses entre los inversionistas del mercado energético y la preocupación justificada de los diseñadores de políticas públicas de los gobiernos, para generar las condiciones de equidad para competir en los mercados, así como evitar el surgimiento de un monopolio poderoso. Sin lugar a dudas, el costo de la electricidad está en función de los precios del combustible con el que genere electricidad, aunado a la tecnología ocupada.

Por esta razón, es importante contemplar la tendencia hacia la baja utilización de los combustibles fósiles, por lo menos del carbón y del crudo. El primero, ha pasado de un 38\% de utilización a nivel mundial en 1965 a un $28 \%$ en 2015, aunque entre 1970 y 2010 osciló su uso entre 25 y 30\%. En cuanto al segundo, el crudo tuvo su mayor pico de uso en la primera mitad de la década de los setenta con cerca del $50 \%$ de su participación en la generación de energía eléctrica, sin embargo su caída ha sido sistemática hasta llegar en 2015 a un 32\%. Por su parte, el gas tiene un crecimiento sostenido desde un 16\% en 1965 hasta un 23\% en el 2015. Mientras que los materiales no renovables y el agua su participación ha sido de un dígito en los últimos cincuenta años. Vale la pena destacar que, los combustibles fósiles, crudo, carbón y gas presentan una tendencia a la baja en los precios de dichos combustibles de enero de 2014 con una proyección de ligera alza para 2016 (WE, 2016).

Narayan (2016) hace un estudio mundial sobre la predicción del consumo energético y el crecimiento económico, la nación mexicana ${ }^{63}$ cae dentro del grupo de la hipótesis del crecimiento del consumo de energía lidera el crecimiento económico y sugiere que esto conlleva a un mayor crecimiento

${ }^{63}$ La Organización para la Cooperación y el Desarrollo Económico (2013) agrupa a 128 naciones, bajo los criterios de: nivel de desarrollo, nivel de ingreso y continente. Con estos elementos, México está en Desarrollo, Ingresos per cápita arriba de la media del Continente Americano. 
económico. La autora, explica la importancia de la energía como un complemento al trabajo y al capital de este argumento unidireccional.

Con base en los resultados de los dos estudios del Banco Mundial WB (2016) y de Narayan (2016), podemos extrapolar el consumo de energía medido en BTU, contra el ingreso per cápita de México que para 2015 era de 9,009.3 dólares a términos reales de 2005, y con eso el cálculo del consumo energético es de 112.38 millones de BTU por persona. Al contrastarlo, con el consumo per cápita global entre 1984-2010 fue de 82.8 millones de BTU con un ingreso per cápita de 9972.8 dólares. Lo anterior, muestra que el país requiere de grandes inversiones en instalaciones de generación eléctrica, ello lleva a un consumo anual de 13.43 Peta BTU ${ }^{64}$.

Ante estos requerimientos energéticos del país, la decisión consensada entre las fuerzas políticas del Partido Revolucionario Institucional, Partido de Acción Nacional, Partido de la Revolución Democrática y Gobierno Federal firmaron el Pacto por México en 2012, a partir de eso se inició un proceso de reformas estructurales, entre ellas la reforma energética, con expectativas muy elevadas, pero en la práctica los resultados han sido cuestionados. En este sentido, valdría retomar a Luhmannn (2005), al comentar la complejidad impone frente a barreras de la capacidad cognitiva y frente a límites de costo de tiempo, las limitaciones de las exigencias de la racionalidad, entendida como la relación entre fines y medios.

Más aun, la complejidad se constituye en los sistemas organizacionales como la relación entre decisiones, calificándose de manera recíproca. Aceptando esta premisa, el análisis de la reforma energética, sus implicaciones en la sociedad mexicana está por verse, hasta la segunda subasta del mercado eléctrico nacional, podemos argumentar que las empresas participantes no son las líderes del mercado, ni tampoco lo son los países potencia en energías renovables e inclusive en la tecnología de ciclo combinado. Para argumentar lo anterior, las dos subastas en el mercado energético, no se observa la participación de los líderes internacionales en el mercado mexicano, dos casos exitosos son Alemania y España de generación de energía renovable (eólica y solar). Por ejemplo, la Unión Europea para 2020 busca producir el 20\% de la electricidad con energía limpia, en el caso específico de Alemania genera el $40 \%$ de la producción mundial a través de la energía eólica (AIE, 2015).

Algo que la propia reforma no contempló las transformaciones culturales de los trabajadores al pasar de una entidad paraestatal a una empresa productiva del estado. Los diseñadores de la reforma energética no

${ }^{64}$ Es el prefijo denominado peta $\mathrm{P} 10^{15}$, equivale a cuatrillones o miles de billones, en este caso se requiere 13.43 cuatrillones de BTU, esta unidad es british thermal unit equivalente a 257 calorías. 
vislumbraron que la Comisión Federal de Electricidad, no sólo es una empresa productiva del estado, una organización burocrática, en palabras de Lourau (2007), señala que las instituciones son estructuras (sistema de roles y posiciones) y mecanismos culturales (convenciones) que son utilizados para regular las relaciones entre los miembros de una sociedad.

Directivos entrevistados coincidieron en comentar que, el personal de la CFE se enfrenta a nuevas dinámicas laborales y a nuevos temores cotidianos. En palabras del coordinador CPT:

Buscamos ser referencia en ingeniería de proyectos termoeléctricos de clase mundial, con un personal especializado en diversas áreas de ingeniería mecánica, eléctrica, civil, electrónica, etcétera. Esto, soporta nuestra organización, somos los pocos del país que hacemos ingeniería completa, nuestro personal está muy especializados y es nuestro principal activo para competir, de manera desafortunada eso no basta, tenemos muchos pendientes que nos ponen en desventaja en el mercado.

Por el lado de los trabajadores, su vida en las organizaciones depende de la habilidad de sentir, cuando una organización sufre modificaciones en su devenir, ésta se manifiesta en la cultura organizacional. Meyerson y Joanne (1987), Aldrich y Ruef (2006), consideran que hay tres formas de presentar las interpretaciones de la cultura organizacional que ha sufrido transformaciones. La primera, consiste en una integración a las nuevas dinámicas desde la alta dirección hasta lo ancho de ella; la segunda es la diferenciación que se da dentro de clúster y se generan etapas multifacéticas, y la tercera es la fragmentación con un proceso transitorio difuso.

En este sentido, es prematuro establecer cuál es el camino que sigue la CFE, pero lo que sí está claro es que los cambios sufridos crean tendencias en la cultura organizacional que mezclan competencias con los productores independientes, entendimiento entre los trabajadores de la empresa productiva del estado y fortalecen o fragmentan su identidad. En palabras de un informante clave comenta lo siguiente: "nos ponen a competir con el mercado energético internacional y no nos das los suficientes recursos, nos estamos pareciendo a Pemex en las dos décadas pasadas, vamos a ser supervisores de compañías, tenemos que aprender a convivir con ellos.”

Esto muestra que, el gobierno federal diseñó la reforma energética con una participación mixta en la cadena de valor del sector. Para ejemplificar, los cambios sufridos dentro de la Secretaria de Energía y las nuevas dependencias creadas (CRE, CENACE, ASEA, CENEGAS Y INEEL, CFE), obedecen a un isomorfismo competitivo, donde hay una lucha por escasos recursos, ello obliga a que las organizaciones adopten estructuras y prácticas eficientes de organizaciones rectoras exitosas (Powell y Dimaggio, 1999). Caso concreto, es la Comisión Reguladora de Energía (CRE), cuya función 
es regular la participación de empresas públicas y privadas, así como garantizar la transparencia en los contratos, permisos y procesos de licitación, todo ello busca el funcionamiento eficiente de los mercados emergentes.

En contraste, Guy (2003), considera que las instituciones no cambian fácilmente, él opina que mantienen un cierto equilibrio, hasta que hay una intermisión que modifique esta situación. Más aun, considera que el rol de los valores que acotan el comportamiento individual es prominente para mantener el equilibrio de las instituciones. Esto, se ejemplifica con la opinión de un informante directivo:

De manera cotidiana nos enfrentamos con colegas del sector privado en diferentes foros $\mathrm{y}$ en algunas actividades del trabajo que coincidimos, nos conocemos, sabemos las capacidades de las empresas, Siemens, Mitsubishi, Iberdrola, General Electric, algunos Consorcios Mexicanos, pero nuestra cultura de calidad es muy sólida, el apego por la Comisión es mucha, ella nos ha dado todo, pero estos cambios son significativos para nosotros, pues nos ponen a competir en condiciones desfavorables.

Al respecto, basta analizar el comportamiento financiero entre $2014 \mathrm{y}$ 2015 para observar grandes problemas de sustentabilidad financiera: el primero de ellos es el impacto negativo en la contratación de pasivos en dólares (PIDIRIEGA y Productores Independientes de Energía), ello provocó una pérdida de más de treinta siete mil millones de pesos. En esa misma orientación, el costo financiero de CFE se incrementó de 2014 a 2015 en un 35\%, lo que significó un monto de 59,357 millones de pesos. En resumen, el total de la deuda de CFE (deuda documentada, PIDIRIEGAS y Contratos de Productores Independientes), en el corto y largo plazo asciende a $432.4 \mathrm{mil}$ millones de pesos a 2015. El 92\% de la deuda es de más de un año y el 56\% de la deuda está contratada en dólares lo cual lesiona seriamente las finanzas de la Comisión (CFE, 2015).

Después de eso, la CFE tiene el pendiente del pasivo laboral, es el 54\% de la deuda de la organización e impacta significativamente en la pérdida neta del estado de resultados. En concreto, el monto es de 630, 371millones de pesos, al pasar de 35,826 jubilados en 2011 a 45, 340 trabajadores en ese estatus en 2015. Todo ello, se enmarca en una plantilla laboral de 88,359 para 2015, de los cuales 47, 962 están en el apartado I (personal reglamentado por el contrato colectivo de trabajo de 2008), cuyo promedio de antigüedad es de 13 años y de 40 años de edad, pese a que hay un poco más de 40 mil empleados en el apartado II (cuenta individual de pensiones (CIJUBILA), el comportamiento futuro del pasivo laboral seguirá teniendo impacto significativo en las finanzas de la empresa productiva del estado. (CFE, 2015) 
$\mathrm{Al}$ respecto un funcionario de alto nivel comentó:

Cuando Don Alfredo Elías Ayub, nuestro director de 1999 a 2011, sale de la institución por cuestiones de salud, considerábamos que había la oportunidad para ocupar el puesto de director de la CFE, teníamos mucha empatía con él porque había liderado a la Comisión de manera firme pero humana. Varios colegas sonábamos para el puesto, el presidente Calderón dirigió la Secretaria de Energía y sabía de nuestro trabajo, pero al saber el nombramiento del nuevo director, me puse a reflexionar y pensé seriamente en la jubilación, después de más de tres décadas entregándome a la institución, concluí que la CFE estaba por sufrir cambios trascendentales.

Por si fuera poco, la CFE ha tenido pérdidas de energía ${ }^{65}$ la última década que oscila entre el 14 y el 17\%, los cuales representan entre el 0.12 y 0.31 del producto interno bruto. Al compararlo con el promedio de los países miembros de la OCDE con 6\% y de los países no miembros de la OCDE con ingresos medios con 9\%, la CFE está muy alejada de esa meta, tan es así que pronostican su índice de pérdida menor al 10\% para 2030. (Jiménez y otros, 2014). Con la misma fuente, es importante señalar que, América Latina y el Caribe son los que presentan el mayor porcentaje de pérdida de todas las regiones del mundo con un 17\%, seguido de África con 14\% y Eurasia con $13 \%$. En contraste, América del Norte y Europa tienen índices de pérdidas similares del $8 \%$. Con todo esto, el panorama del sector eléctrico mexicano para la empresa productiva del estado es bastante complejo.

Conclusiones

Derivado de este recorrido analítico por tres áreas estratégicas del sector y por cifras globales de la CFE, se está en posibilidad de llegar a varias conclusiones que fijan un posicionamiento sobre la competitividad y sustentabilidad de dicha empresa productiva del estado, las cuales se relaciona a continuación.

El diseño organizacional de las instituciones requiere de tiempo para demostrar su eficiencia, al momento pareciera un conglomerado de instituciones y regulaciones que no perfilan organizaciones articuladas. En forma particular, la Comisión Reguladora de Energía en un futuro se enfrentará con diversos conflictos sociales, en términos del uso de la tierra y recursos naturales para generar energía, los cuales eventualmente pueden ser cuestionados por las organizaciones sociales. Además, el Centro Nacional de Control de Energía y el Sistema Eléctrico Nacional deberá seguir criterios de

${ }^{65}$ Estas pérdidas de energía se dan en toda la cadena de valor, desde la generación, transmisión, distribución y facturación, aunque en el caso de México el mayor porcentaje de pérdida es en la distribución. Aunque se clasifican en pérdidas de electricidad técnicas (fijas y variables) y no técnicas (robo, fraude consumo no contabilizado y problemas de gestión (BID, 2013). 
disponibilidad de energía con productores independientes, cuyo motivo principal es la utilidad, en ese sentido se pone en riesgo la cobertura a zonas desfavorecidas.

Dentro de los obstáculos serios con los que debe lidiar la CFE son sus pasivos, por un lado los acreedores de largo plazo negociaron sus contratos en dólares y con tasa variables, ello ha repercutido significativamente en sus estados financieros. Aunado a esto, el pasivo laboral corroe las finanzas de la institución, la masa de jubilados se incremente gradualmente y las condiciones futuras son adversas. Ello es un lastre que no le permite contender en igualdad de condiciones en un mercado eléctrico muy competido con participación nacional e internacional. Pese a esto, el futuro es más incierto, pues los grandes luchadores no entran a escena todavía, lo que resta posibilidad de mantener los porcentajes de participación en el mercado, es más su caída será sistemática.

La alta especialización del personal y una cultura sólida de calidad es un positivo de la CFE, el conocimiento acumulado y la diferenciación de los recursos humanos es una ventaja competitiva que le puede permitir hacer alianzas estratégicas con competidores nacionales e internacionales y así mantenerse como un actor relevante en el sector. Más aun, los directivos de la institución tendrán que ser innovadores en la comercialización del conocimiento acumulado, cogenerando valor con sus competidores para fortalecer su posición.

En suma, las condiciones contextuales son desfavorables para la CFE, el presupuesto y la participación en el mercado disminuyen; los pasivos y la competencia aumentan. La empresa productiva del estado está gravemente enferma, no hay curas mágicas, pero es recomendable cuidar sus recurso y enfocar en sus virtudes sus esfuerzos, no pelear en muchos frentes, seleccionar un portafolio de generación que lo inserte en la competencia del sector.

\section{References:}

1. Aldrich, H. and Ruef, M (2006). Organizations Envolving, $2 a$ edition. London: Sage Publications.

2. Bauer, M y García, L. Coordinadores. (2008). Energía en México el Arranque del siglo XXI, realidades y opciones. México: El Colegio Nacional.

3. Beck, U. (2002). La sociedad del riesgo global. Madrid: Siglo XXI de España editores.

4. Beltrán, M. (2000). Cinco vías de acceso a la realidad social, en García, M., Ibáñez, J. y Alvira F. (2000). El análisis de la realidad social. Métodos y técnicas de investigación, tercera edición revisada: Madrid. Alianza editorial. 
5. Bogdan, R., y Taylor, S. (2000). Introducción a los métodos cualitativos de investigación. España: Paidos Básica.

6. Finley, M. (2016). Informe Estadístico Mundial de BP sobre Energía. London: BP World Energy.

7. Goldstein, A. (2009). Multinational Companies from Emerging Economies. Great Britain: Palgrave MacMillan.

8. Guy, B (2003). El nuevo institucionalismo. Barcelona: Gedisa.

9. Hernández S.R, Fernández C.C, Baptista L.P (2005). Fundamentos de metodología de la investigación. México: McGraw Hill, Interamericana.

10. Jiménez, R., Serebrisky, T., y Mercado, J (2014). Electricidad Perdida. Washington: Banco Interamericano de Desarrollo.

11. Lourau, R. (2007). El análisis institucional. Argentina: Amorrortu.

12. Luhmann, N. (1998). Complejidad y Modernidad: de la unidad a la diferencia. Valladolid: Editorial Trotta.

13. Luhmann, N. (2005). Organización y decisión: Autopoiesis, acción y entendimiento comunicativo. Barcelona: Anthropos y UIA.

14. Meyerson, D. and Joanne, M. (1987). Culture Change: An integration of three diferent views. Journal of Management Studies. 24, 6 (November), pp. 623-647.

15. Narayan, S. (20016). Predictability within the energy consumption economic growth nexus: Some evidence from income regional groups. Economic Modelling. April 54 (2016), pp. 515-521

16. Pace, S. (2004). A grounded Theory of the flow experience of Web users. Intentional Journal of Human-Computer Studies, 60 (3), pp. 327-363.

17. Pacheco, A. y Cruz, C. (2006). Metodología Crítica de la Investigación. México: Editorial CECSA.

18. Powell, W. y Di Maggio, P. (2001). El Nuevo institucionalismo en el análisis organizacional. México: FCE.

19. Quintanilla, A. y Arfeuille, G. (2011). Potencial eléctrico de los recursos energéticos renovables en México en María González Ávila, E., Muñoz Meléndez G. y Ortega Rubio A., (coords.). Hacia la sustentabilidad ambiental de la producción de energía en México. Baja California Sur: Colegio de la Frontera Norte.

20. Rodríguez, J.M (2006). Estrategias Energéticas para el Sector Eléctrico, CFE, CPT. Material sin publicar.

21. Salvadore, D. (2010). International Economics. Tenh edition. USA:Wiley.

22. Stake, R. (2005). Investigación con estudio de casos. España: Ediciones Morata, S.L.

23. Páginas electrónicas 
24. CENEGAS (2016). PLAN QUINQUENAL DE EXPANSIÓN DEL SISTEMA DE TRANSPORTE Y ALMACENAMIENTO NACIONAL INTEGRADO DE GAS NATURAL 2015-2019, disponible en http://www.gob.mx/cms/uploads/attachment/file/116777/511_DGGN P_260_16_OT_11_Primera_Revisi_n_PQ.pdf, consultado en agosto de 2016.

25. Comisión Federal de Electricidad. Informe anual de la CFE, 2011, 2012, 2013, 2014, 2015. Disponible en www.cfe.gob.mx

26. Diario Oficial de la Federación (DOF) (1994, 2014, 2016).

27. Monitoreo Energético. http://www.monitorenergetico.com.mx/, consultada en abril de 2016

28. Ocde/aie (2015). World Energy Outlook. International Energie Agency. París: IEA PUBLICATIONS, disponible en www.worldenergyoutlook.org, consultado en abril de 2016.

29. Secretaría de Energía http://www.gob.mx/sener/articulos/empresas-ganadoras-de-la$(\mathrm{SE})$, primera-subasta-de-largo-plazo, consultado en abril de 2016.

30. United Nations Population Fund, UNFPA Annual Report, 2015. http://www.unfpa.org/sites/default/files/pub-

pdf/UNFPA_2015_Annual_Report.pdf, consultado en Agosto de 2015.

31. World Bank. http://www.worldbank.org/

32. World Bank, http://datos.bancomundial.org/indicator/NY.GDP.MKTP.KD.ZG, consultado en agosto de 2016. 